\title{
Extranodal Natural Killer/T-Cell Lymphoma, Nasal Type, with Primary Manifestation as an Upper Eyelid Swelling
}

\author{
Akiko Kanzaki ${ }^{1}$, Yoko Funasaka ${ }^{1}$, Munenaga Nakamizo ${ }^{2}$, Ayaka Shima ${ }^{3}$, \\ Takeshi Ryotokuji ${ }^{3}$, Kazuo Dan ${ }^{3}$, Mika Terasaki ${ }^{4}$, Yuichi Sugisaki ${ }^{4}$, \\ Yu Fukuda ${ }^{4}$, Seiji Kawana ${ }^{1}$ and Hidehisa Saeki ${ }^{1}$ \\ ${ }^{1}$ Department of Dermatology, Nippon Medical School, Tokyo, Japan \\ ${ }^{2}$ Department of Otorhinolaryngology, Nippon Medical School, Tokyo, Japan \\ ${ }^{3}$ Division of Hematology, Department of Internal Medicine, Nippon Medical School, Tokyo, Japan \\ ${ }^{4}$ Department of Analytic Human Pathology, Nippon Medical School, Tokyo, Japan
}

\begin{abstract}
Extranodal natural killer/T-cell lymphoma (ENK/TCL) is most often in the nose or the nasopharynx but can present elsewhere. We report a rare case of ENK/TCL that presented as swelling of an upper eyelid without ocular involvement. A 76-year-old man visited our hospital with a swollen lesion of the left upper eyelid which had appeared 2 months earlier. A biopsy of the upper eyelid revealed slight perivascular and periadnexal infiltration of mononuclear cells with dermal edema. Treatment with oral prednisolone at a dosage of $20 \mathrm{mg} /$ day decreased the eyelid swelling. However, 5 months later, exacerbation of the swelling and nasal congestion were observed. A second biopsy of the upper eyelid revealed a diffuse dermal infiltrate composed of mononuclear cells with an angiocentic growth pattern. Immunohistochemical studies and in situ hybridization showed natural killer-lineage antigens (CD56, granzyme B, and T-cell intracellular antigen 1) with expression of Epstein-Barr virus. These findings lead to the diagnosis of ENK/TCL. We treated the patient with radiation therapy (50 Gy) and 3 courses of a regimen including dexamethasone, carboplatin, etoposide, and ifosphamide. This case suggests that ENK/TCL can present with swelling of an upper eyelid as the primary sign of the skin lesion. Swelling of an upper eyelid should be considered in the differential diagnosis of ENK/TCL.
\end{abstract}

(J Nippon Med Sch 2016; 83: 177-179)

Key words: extranodal natural killer/T-cell lymphoma, nasal type, eyelid swelling

\section{Introduction}

Extranodal natural killer/T-cell lymphoma (ENK/TCL) usually occurs in the nose and nasopharyngeal region, but extranasal and disseminated disease can occur. Several cases of ENK/TCL have been reported to involve ocular lesions ${ }^{1-4}$. We report a rare case of ENK/TCL that presented as the initial skin involvement of swelling of an upper eyelid without ocular symptoms.

\section{Case Report}

A 76-year-old man visited our hospital with a swollen lesion on the left upper eyelid which had appeared 2 months earlier (Fig. 1-a). Baseline blood chemistry results and blood counts were normal. A biopsy of the edematous erythematous lesion of the upper eyelid revealed slight perivascular and periadnexal infiltration of mononuclear cells with dermal edema (Fig. 1-b, c). Oral predonisolone administered at a dosage of $20 \mathrm{mg} /$ day decreased eyelid swelling; however, 5 months later, exacerbation of the swelling and nasal congestion were observed (Fig. 1-e). A second biopsy of the upper eyelid revealed a diffuse mononuclear cell infiltration with an angiocentric growth pattern (Fig. 1-f). The small and medium-sized mononuclear cells showed moderate nuclear atypia (Fig. 1-g). Immunohistochemical studies were positive for antibodies against $\mathrm{CD} 3, \mathrm{CD} 56$, gran-

Correspondence to Akiko Kanzaki, MD, Department of Dermatology, Nippon Medical School, 1-1-5 Sendagi, Bunkyo-ku, Tokyo 113-8602, Japan

E-mail: konchi@nms.ac.jp

Journal Website (http://www.nms.ac.jp/jnms/) 

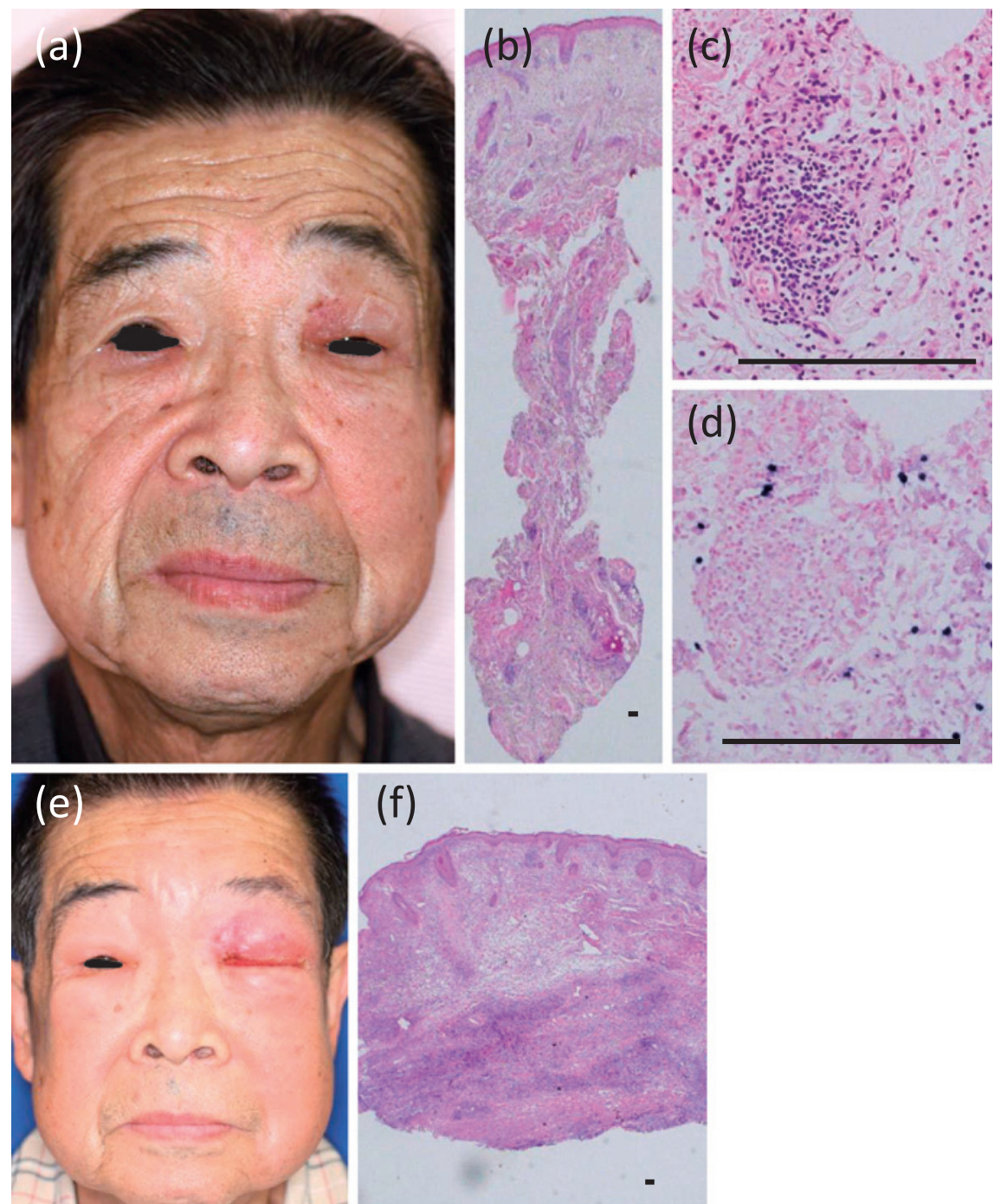

(f)
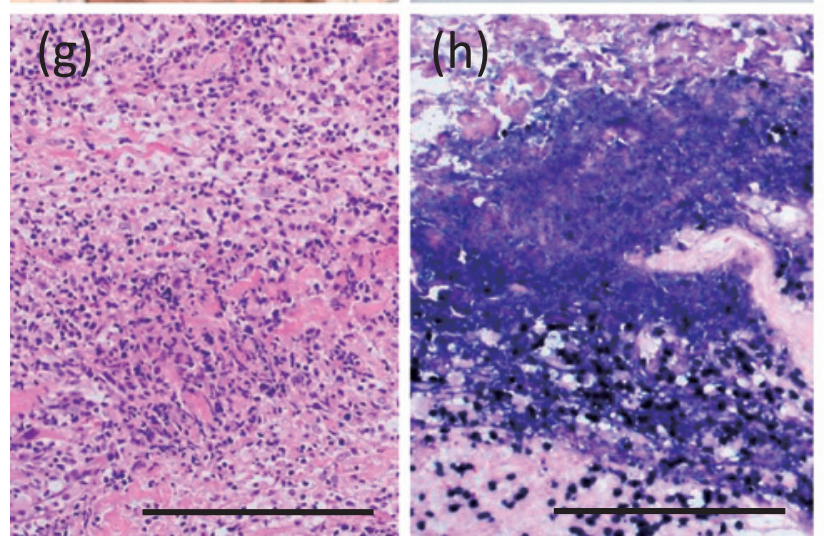

Fig. 1 (a) Clinical features during the patient's first visit to our hosptial. Physical examination showed swelling of the left eyelid. (b, c) Results of the first biopsy. The skin specimen showed slight perivascular and periadnexal mononuclear infiltration with dermal edema (b: $\times 20, c: \times 400)$. (d) Re-examination of the eyelid specimen of the first biopsy. Most perivascular small mononuclear cells in the subcutaneous area were positive for Epstein-Barr virus-encoded small RNA (in situ hybridization), although the infiltration was minimal. $(\times 400)(\mathrm{e})$ Clinical features at 5 months after the initial visit. (f, g) Results of the second biopsy. The skin biopsy specimen showed angiocentric dense infiltration of mononuclear cells with nuclear atypia in the dermis $(\mathrm{f}: \times 20, \mathrm{~g}: \times 400)$. (h) The pleomorphic lymphocytes showed positivity for Epstein-Barr virus-encoded small RNA (×400). Scale bars: $100 \mu \mathrm{m}$. 
zyme B, and T-cell intracellular antigen 1 but not against CD20. The staining pattern of CD3 was cytoplasmic. In situ hybridization for Epstein-Barr virus (EBV)-encoded small RNA (EBER) was positive (Fig. 1-h). The biopsy of an intranasal necrotic mass obtained histological findings extremely similar to those of the second biopsy of the upper eyelid. Re-examination of the eyelid specimen obtained at the first biopsy showed perivascular infiltration of small cells positive for CD56 and EBER in the subcutaneous area (Fig. 1-d). On the basis of these findings, ENK/TCL, nasal type, was diagnosed. The oropharyngeal cancer that the patient had had 1 year before he first visited our hospital had led to the same diagnosis of ENK/TCL by the re-examination. Radiation therapy (50 Gy) and 3 courses of a regimen including dexamethasone, carboplatin, etoposide, and ifosphamide were administered. Positron emission tomography and computer tomography showed complete remission, but pancytopenia and dysphagia, due to the recurrence of ENK/ TCL in the upper oropharyngeal area, appeared later.

\section{Discussion}

Patients who have ENK/TCL nasal type present with symptoms of nasal obstruction or epistaxis due to the mass lesion or with extensive, destructive midfacial lesions. The lymphoma can extend to adjacent tissues, such as the nasopharynx, paranasal sinuses, orbit, oral cavity, palate, and oropharynx. At presentation the disease is usually in the upper aerodigestive tract. Skin lesions of ENK/TCL are described as commonly nodular, often with ulceration ${ }^{5}$.

Lymphoma associated with EBV infection is found in 2 groups of patients: those aged 13 to 29 years and those who are older than 50 years. In the younger group the lymphoma is reportedly associated with chronic, active EBV infection. Furthermore, when lymphocytes of EBERpositive cells have been observed to infiltrate deeply in the skin of these younger patients, the risk of lymphoma is increased and eyelid swelling is sometimes observed. In the older group of patients, the lymphoma is believed to directly extend or metastasize from the nose to other skin lesions. Because of direct invasion, midfacial involvement is easily observed. In the present case, ENK/ TCL was difficult to diagnose with the initial biopsy because only a small number of lymphoma cells had infiltrated the skin of the eyelid.

The Lymphoma Study Group of the Japan Clinical Oncology Group reported a phase I/II study of concurrent chemoradiotherapy for localized ENK/TCL, nasal type. The 5-year overall survival rate was $70 \%$ for patients who had undergone radiotherapy and received a 2/3 dose of a regimen including dexamethasone, carboplatin, etoposide, and ifosphamide 5 . We had treated our patient in the same way to control the development of the disease.

The largest cohort of ENK/TCL with ocular and adnexal involvement has been reported by Woog et al: 4 of the 8 patients (50\%) with ENK/TCL involving the orbit or ocular adnexa had systemic involvement at presentation, 5 of the $8(62.5 \%)$ had concurrent sinonasal involvement, and 3 patients $(37.5 \%)$ had only orbital involvement ${ }^{6}$. Although nasal symptoms were not initially noted and visual problems were absent, this type of lymphoma should also be considered when abnormal eyelid swelling is noted.

In conclusion, we reported a case of ENK/TCL that presented as the initial skin involvement of swelling of an upper eyelid without ocular symptoms in the aged man. Because the diagnosis was difficult, the image of such a variant should be considered by dermatologists.

Conflict of Interest: The authors declare no conflict of interest.

\section{References}

1. Ely A, Evans J, Sundstrom JM, Malysz J, Specht CS, Wilkinson M: Orbital involvement in extranodal natural killer T cell lymphoma: an atypical case presentation and review of the literature. Orbit 2012; 31: 267-269.

2. Yoo JH, Kim SY, Jung KB, Lee JJ, Lee SJ: Intraocular involvement of a nasal natural killer T-cell lymphoma: a case report. Korean J Ophthalmol 2012; 26: 54-57.

3. Lee EJ, Kim TW, Heo JW, Yu HG, Chung H: Natural killer/T-cell lymphoma of nasal type with intraocular involvement: case report. Eur J Ophthalmol 2010; 20: 215217.

4. Chan JKC, Quintanilla-Martinez L, Ferry JA, Peh SC: Extranodal NK/T-cell lymphoma, nasal type. In World Health Organization Classification of Tumours: Pathology and Genetics of Tumours of Haematopoietic and Lymphoid Tissues (Jaffe ES, Harris NL, Stein H, Vardiman JW, eds), 2008; pp 285-288, IARC Press, Lyon, France.

5. Yamaguchi M: Development of new treatments for extranodal NK/T-cell lymphoma in Japan. Rinsho Ketsueki 2015; 56: 639-644.

6. Woog JJ, Kim YD, Yeatts RP, Kim S, Esmaeli B, Kikkawa D, Lee HB, Korn BS, Punja K, Habermann TM, Colgan JP, Salomao D, Cameron JD: Natural killer/T-cell lymphoma with ocular and adnexal involvement. Ophthalmology 2006; 113: 140-147.

(Received, January 12, 2016)

(Accepted, March 24, 2016) 